\title{
Development of the EQ-5D-Y: a child-friendly version of the EQ-5D
}

\author{
Nora Wille · Xavier Badia $\cdot$ Gouke Bonsel $\cdot$ Kristina Burström • \\ Gulia Cavrini · Nancy Devlin · Ann-Charlotte Egmar - Wolfgang Greiner • \\ Narcis Gusi $\cdot$ Michael Herdman · Jennifer Jelsma $\cdot$ Paul Kind • \\ Luciana Scalone $\cdot$ Ulrike Ravens-Sieberer
}

Accepted: 21 March 2010/Published online: 20 April 2010

(C) The Author(s) 2010. This article is published with open access at Springerlink.com

\begin{abstract}
Purpose To develop a self-report version of the EQ-5D for younger respondents, named the EQ-5D-Y (Youth); to test its comprehensibility for children and adolescents and
\end{abstract}

to compare results obtained using the standard adult EQ-5D and the EQ-5D-Y.

Methods An international task force revised the content of EQ-5D and wording to ensure relevance and clarity for
N. Wille · U. Ravens-Sieberer $(\bowtie)$

Department of Psychosomatics in Children and Adolescents, Research Unit Child Public Health, University Medical Center Hamburg-Eppendorf, Martinistr. 52, 20246 Hamburg, Germany e-mail: ravens-sieberer@uke.de

\section{Badia}

Health Economics \& Outcomes Research,

IMS Health, Doctor Ferran 25-27,

2, 08034 Barcelona, Spain

\section{G. Bonsel}

Department of Prenatal Medicine and Obstetrics (Location Woudenstein, L3-060), Erasmus Medical Centre, Institute of Health Policy \& Management, P.O. Box 1738, 3000 DR Rotterdam, The Netherlands

\section{K. Burström}

Department of Learning, Informatics, Management and Ethics, Medical Management Centre, Karolinska Institutet,

Nobels väg 15a, 17177 Stockholm, Sweden

\section{K. Burström}

Department of Public Health Sciences,

Division of Social Medicine, Karolinska Institutet,

Nobels väg 15a, 17177 Stockholm, Sweden

\section{G. Cavrini}

Department of Statistics, University of Bologna,

Via delle Belle Arti 41, 40126 Bologna, Italy

\section{N. Devlin}

Office of Health Economics \& Senior Associate, King's Fund, 12 Whitehall, London SW1A2DY, UK

\section{A.-C. Egmar}

The Red Cross University College, Box 55676,

10215 Stockholm, Sweden

W. Greiner

Department for Health Economics and Health Care

Management, University of Bielefeld, School of Public Health, P.O. Box 1001 31, 33501 Bielefeld, Germany

N. Gusi

University of Extremadura, Faculty of Sport Sciences, Avda Universidad, 10071 Caceres, Spain

M. Herdman CIBER en Epidemiología y Salud Pública (CIBERESP), Barcelona, Spain

\section{Herdman}

Health Services Research Unit, IMIM-Hospital del Mar, Unitat de Recerca en Serveis Sanitaris, Parc de Recerca Biomedica de Barcelona, Doctor Aiguader, 88, 08003 Barcelona, Spain

\section{J. Jelsma}

Division of Physiotherapy, University of Cape Town, School of Health and Rehabilitation Sciences, Anzio Road Observatory, Cape Town 7925, South Africa

\section{P. Kind}

Centre for Health Economics, Alcuin College,

University of York, York YO10 5DD, UK

\section{Scalone}

Department of Clinical Medicine and Prevention, Research Centre On Public Health, University of Milano - Bicocca, Villa Serena, Via Pergolesi 33, 20052 Monza, Italy 
young respondents. Children's and adolescents' understanding of the EQ-5D-Y was tested in cognitive interviews after the instrument was translated into German, Italian, Spanish and Swedish. Differences between the EQ-5D and the EQ-5D-Y regarding frequencies of reported problems were investigated in Germany, Spain and South Africa.

Results The content of the EQ-5D dimensions proved to be appropriate for the measurement of HRQOL in young respondents. The wording of the questionnaire had to be adapted which led to small changes in the meaning of some items and answer options. The adapted EQ-5D-Y was satisfactorily understood by children and adolescents in different countries. It was better accepted and proved more feasible than the EQ-5D. The administration of the EQ-5D and of the EQ-5D-Y causes differences in frequencies of reported problems.

Conclusions The newly developed EQ-5D-Y is a useful tool to measure HRQOL in young people in an ageappropriate manner.

Keywords Child health · Adolescent health - HRQOL · Measurement · EQ-5D

$\begin{array}{ll}\text { Abbreviations } \\ \text { HRQOL } & \text { Health-related quality of life } \\ \text { VAS } & \text { Visual analogue scale } \\ \text { EQ-5D-Y } & \text { EQ-5D youth version }\end{array}$

\section{Introduction}

Health-related quality of life (HRQOL) is increasingly considered an important area of research in both children and adolescents (here defined as persons aged 8 to 11 and 12 to 18, respectively). The concept of HRQOL focuses on the viewpoint of the individual and is factored into different areas of health assessment, such as the impact of disease on daily living and functioning or health care utilization [1-3]. Studies in this field require measurement procedures appropriate to the development stage of the respondent [4-6]. As a response to this need, several instruments to assess generic and disease-specific HRQOL in children and adolescent have been developed. These are applied in epidemiological surveys, clinical studies, quality assurance and health care performance assessment [1, 2, 6-11].

The most extensive approach to the development of HRQOL instruments for young respondents includes qualitative pilot studies in order to identify child- and adolescent-specific domains of HRQOL and derive item statements from interviews with young respondents and parents [12]. This approach may be favourable from a theoretical point of view [1] and has been used to develop a variety of questionnaires (e.g. [13-16]). Most instruments, however, were developed by experts based on reviews of the literature and other instruments, expert opinion, informal or systematic parental enquiries, or clinical experience, with little reference to the views of the targeted population (e.g. [17-19]). A last approach is to modify adult HRQOL instruments so that they are suitable for younger respondents. Although there is a consensus that the specific developmental aspects of childhood and adolescence should be represented in HRQOL instruments, previous research has shown that it is possible to measure HRQOL in younger respondents using the same dimensions as in adult instruments and even the same items [1]. Furthermore, the three main components of HRQOL in adults (physical, psychological and social) are clearly of relevance in younger respondents, too. Nevertheless, the specific issues addressed by items and the way they are worded need to be tailored to the target population. Where this is achieved, the development of questionnaires for younger respondents by modifying adult questionnaires facilitates comparisons of HRQOL in adults and younger populations. This might be particularly useful in cohort studies investigating HRQOL in severe childhood chronic conditions that last into adult life, such as cerebral palsy or epilepsy. It is also especially important to be able to track changes in HRQOL across the life course in progressive conditions such as cystic fibrosis.

The EQ-5D is a widely used standardized generic measure of HRQOL [20] originally designed for use in adult populations aged 18 and over. It has been utilized internationally in many settings, such as clinical trials and population surveys. Its dissemination is supported by its concise contents, its applicability to a wide range of health conditions and its twofold reporting format, both as a descriptive profile and as an index which can be interpreted as a preference value. Although the literature emphasizes the use of EQ-5D in economic evaluations, it can nevertheless also be used as a measure of health status in general (non-economic) health assessments. Against the background of the increasing importance of measuring HRQOL in children and adolescents, there has been a demand by end-users of the EQ-5D for a well-validated version for younger respondents. The main rationale of such a version would be to enable young patients and populations to self-report their health, where data might otherwise require collection by proxy. Given the benefits of the conventional EQ-5D, there are a wide range of possible applications for a version for younger respondents, including population health surveys, routine measurement and monitoring in health care settings, and use in clinical research and practice.

Hennessy and Kind [21] reported adequate performance of the EQ-5D in adolescents aged 12 to 18 but pointed out 
the need to ensure that the language and concepts embodied in the instrument are correctly understood by young respondents. Use of EQ-5D in younger age groups has since been investigated in several countries [22-27]. These studies investigated the performance of the instrument in young people from a quantitative point of view but also conducted several extensive qualitative assessments to explore respondent perceptions of the instrument and their ability to comprehend and complete it. From early 2006, the accumulated body of research was considered sufficient to be able to integrate the results and develop a version of the EQ-5D for young people. For this version, a lower age limit of 8 was chosen, as it was not considered possible to rely only on child self-report below this age.

The present paper describes the development of the EQ$5 \mathrm{D}-\mathrm{Y}$ with regard to the phrasing of the modified questionnaire, the translation procedure and qualitative testing of the new version. Results of a quantitative comparison between the standard adult EQ-5D and the EQ-5D-Y are also presented. Results from its validation in an international study and extensive discussion of its use in possible economic valuation will be provided elsewhere [28].

\section{Methods}

\section{EQ-5D}

The standard adult EQ-5D consists of a descriptive system that comprises five items referring to the domains mobility, self-care, usual activities, pain/discomfort and anxiety/ depression scored as presenting no problems, moderate problems or severe problems. In addition, respondents use a vertical, graduated Visual Analogue Scale (VAS) to rate their own health between 0 (the worst) and 100 (the best health state he/she can imagine). Value sets elicited from general population samples are available to convert profiles derived from the 5-dimensional descriptive system into a weighted health state index [29].

\section{Modification process}

Teams from seven countries (Germany, Italy, South Africa, Spain, Sweden, The Netherlands, United Kingdom) participated in the development of the EQ-5D-Y within the framework of a task force on behalf of the EuroQol-Group. The 13 members of the task force were all experienced in quality of life research and members had additional specific expertise in child psychology, paediatrics, health economics, statistics, sport sciences and/or rehabilitation sciences. The development process included five steps: (1) consideration of the EQ-5D's five domain definitions by the expert committee to determine applicability to a younger age group, (2) revision of questionnaire wording to optimize item comprehension for younger respondents, (3) translation, (4) cognitive interviews, (5) integration of results and decision-making on harmonization into a provisional new questionnaire and (6) comparison of results between the modified version for young respondents and the standard adult EQ-5D.

(1) Revision of domain definitions: Definitions of the conceptual domains underlying the EQ-5D's five items and the VAS are available [30]. The definitions clearly specify the domain which each item is intended to cover and help to ensure consistent interpretation of the questionnaire by, for example, researchers and translators. In the first stage of development, these definitions were used as a model to develop comparable definitions for the EQ-5D-Y. The task force reviewed the definitions of the EQ-5D concepts taking into account the developmental stages of children and adolescents. The focus of the review was on the suitability of the implied item content to younger respondents' HRQOL. As the intention was to develop an instrument that would be comparable with the EQ$5 \mathrm{D}$, the additional inclusion of child- and adolescentspecific domains was not considered. Partially modified definitions of the EQ-5D concepts were adopted for the EQ-5D-Y and provided a guideline for cognitive interviews with children and adolescents.

(2) Revision of wording and layout: In the second stage of the development process, questionnaire wording (including instruction, headings and response options) was modified to improve comprehension and to reflect the adapted domain definitions developed at stage (1). Previous experience with administering the EQ-5D in young respondents as well as results from previous qualitative assessments was taken into account. Where necessary, dimension definitions were further modified to reflect changes in questionnaire wording.

At the end of the process, a pilot version of the EQ-5D-Y was agreed on.

(3) Translation: The EQ-5D-Y pilot version was translated according to the EuroQoL translation methodology into languages in which cognitive debriefing would be performed [31]. First, two independent forward translations were performed by native speakers of each target language who were fluent in English and who had experience in the translation of HRQOL measures. Secondly, the translators and a local task force member assessed the forward translations in terms of their conceptual equivalence with the original and developed a consensus version in the 
target language. Thirdly, the consensus version was translated back into English by a native English speaker. Finally, the translation process was reviewed by members of the project team and at least one of the translators to resolve any remaining problems and to generate the final consensus version.

(4) Cognitive interviews: In Germany, Italy, Spain and Sweden trained interviewers from the project teams administered the EQ-5D-Y to convenience samples of healthy and chronically ill young people aged 8 to 18 . After self-completing the questionnaire, respondents participated in cognitive interviews to investigate its comprehensibility, possible misinterpretations and acceptance. Their understanding of the different items was compared with the previously agreed definitions of EQ-5D-Y dimensions. The cognitive interviews were conducted using several techniques [32, 33]. In order to gain maximal information, different methods were employed. Each country employed either the Paraphrasing or the General Probing technique in order to test whether the instrument was comprehensible in each language version. In the Paraphrasing Method, interviewees were asked to repeat each item in their own words to determine whether the respondent understood the item in the manner intended [34]. The General Probing Method [35] was used to question interviewees as to whether the item was comprehensible and clear. Respondents were also asked to suggest alternative or better item descriptors. In Italy and Spain, respondents were asked to judge item and overall questionnaire comprehensibility on a scale of 0 to 10 using the Understanding Numeric Scale Method [36]. In Italy and Sweden, a free association approach was followed to primarily investigate semantic aspects of the instrument [37]. Subsequently, adjustments were made to the questionnaire, resulting in a second final consensus version in each language. In Sweden, part of the work [24] was performed before the task force was formed, hence, within the scope of the task force the development procedure was finalized [38, 39].

(5) Integration and decision-making on harmonization: After revision and discussion of the pre-test results from different countries, the English language EQ$5 \mathrm{D}-\mathrm{Y}$ source version was approved by the development team and made available for further testing in larger samples. Results of that testing are described elsewhere [28].

(6) Comparison of the EQ-5D and the EQ-5D-Y: Finally, in order to compare the results obtained with the EQ5D-Y and the EQ-5D, both versions were administered to school-based samples in Germany, Spain and South Africa. In Germany, pupils aged 10 to 18 (mean $=13.9$ years; $\mathrm{SD}=1.8)$ from 23 randomly selected elementary and high schools in Berlin randomly received either the EQ-5D-Y $(n=756)$ or the EQ-5D $(n=730)$. Similarly, in South Africa, pupils aged 13 to 19 (mean $=15.5$ years; $S D=1.3)$ from an English high school in a middle-class area of Cape Town filled in either the EQ-5D-Y $(n=258)$ or the EQ-5D $(n=259)$ after random allocation of the versions [40]. In Spain, 973 pupils aged 8 to 18 (mean $=13.0$ years; $\mathrm{SD}=2.7$ ) attending one of six schools in Caceres received a test battery which included the EQ-5D-Y at the beginning and the EQ$5 \mathrm{D}$ at the end. Standardization of the sample sizes in each country was not possible as resources and local situations differed. However, all samples were balanced in terms of gender distribution (between $49.7 \%$ and $50.8 \%$ boys) and had similar social and healthrelated characteristics. The majority of German (91.1\%) and Spanish (94.4\%) respondents reported average or above average family wealth. South African questionnaires did not assess perceived family wealth, but pupils came from an upper middle-class social background. In all countries, the majority of respondents (range $83.4 \%-94.5 \%$ ) reported good, very good or even excellent health and the percentage of pupils taking medication for a chronic illness ranged between $11.0 \%$ and $15.9 \%$. Frequencies of reported problems on the EQ-5D and EQ-5D-Y were compared using $\chi^{2}$ statistics in Germany and South Africa and using the Wilcoxon signed-rank test in Spain. A minimum number of $n=200$ respondents per questionnaire version was required to detect differences of 0.25 a standard deviation with $\alpha=0.05$ and a power of $80 \%$. The percentage of missing values on the two versions was compared using $\chi^{2}$ in Germany and South Africa and using the McNemar test in Spain. In all countries, only pupils who were present on the day the study was conducted completed the questionnaires during school time.

There were small differences with regard to the treatment of VAS scores. In Germany and South Africa, VAS scores were counted as missing when the standard adult EQ-5D VAS was not filled out correctly i.e. when respondents did something other than drawing a line from the box with the words 'your health today' to their chosen VAS score. In Spain, all VAS scores were accepted as valid as long as they were unambiguous even when the standard method was not followed (e.g. when a pupil used the VAS as a thermometer, by drawing a line from the bottom to the score).

In all countries, informed consent from parents or guardians of the pupils was a pre-condition for participation 
in the cognitive interviews or the quantitative testing. In line with national regulations, permission to collect data was obtained from the data protection commissioner in charge (Germany, Italy, Spain) or the appropriate ethics committee (Sweden: Karolinska Institutet Number 2006/1534-31/2, South Africa: Medical Research Ethics Committee of the University of Cape Town and South African Department of Education, Italy: ethics committees of the participating hospitals).

\section{Results}

Revision of EQ-5D domain concepts and child-friendly adaptations

The results of the revision of domain concepts are summarized in Table 1. There was consensus that the overall concept of 'health' applies to younger age groups as well and that the five EQ-5D dimensions were all important domains for child and adolescent HRQOL. However, some minor adaptations were introduced in their operationalization to clarify the meaning of these dimensions for younger respondents. In general, it was considered important to clarify that items refer exclusively to healthrelated impairment and not to age-related difficulties. In particular, it was considered important to point out that reports of problems in performing self-care activities do not imply impairment if the degree of help is appropriate for respondents of that particular age and cultural background. Other modifications included the use of more appropriate examples in the 'Usual activities' dimension. In the domains 'Anxiety/Depression' as well as 'Pain/ Discomfort', no alteration or explanation of content was deemed necessary. Finally, the concept of the VAS was also considered applicable in children and adolescents.

Revision of EQ-5D wording and changes in the EQ-5D-Y

After reviewing the EQ-5D domain concepts for applicability in children and adolescents, the wording of the questionnaire was reviewed to enhance comprehensibility and potentially improve data quality. Uniform answer options were provided for four domains indicating 'no', 'some' and 'a lot of' problems. In the 'Anxiety/Depression' domain 'not', 'a bit' and 'very' were chosen as the corresponding wording. To facilitate response selection the qualifiers were underlined in each response option. The modification of the quantifiers resulted in a difference with the original EQ-5D in the 'Mobility', 'Self-Care' and 'Usual activities' domains, where the highest level of severity refers to an inability to perform the activities in question. The rephrasing to 'a lot of problems' in the EQ5D-Y implies less extreme statements and required adjustments in the items' domain definitions. Furthermore, modification of the response options in the 'Self-Care' dimension (EQ-5D-Y: 'Looking after myself') by inserting the examples 'washing and dressing' restricts item content to activities of personal hygiene in the EQ-5D-Y. Thus, the items explanation in the domain's definition had to be slightly adapted. However, the majority of wording changes to facilitate comprehension did not affect item content.

Regarding an adaptation of the VAS for younger respondents the national teams tested different variants in their pilot tests, collected data on their performance and agreed on the best solution afterwards. The final version of the EQ-5D-Y is presented in Table 2 which also shows the new wording of the EQ-5D-Y VAS.

\section{Results of the cognitive interviews}

The sample characteristics of the respondents included in the cognitive interviews conducted in Germany, Italy, Spain and Sweden are shown in Table 3.

The young people were generally positive about the questionnaire and broadly accepted its general structure. Almost all interviewees were able to respond to the items without assistance. In particular, the 'Mobility (walking about)', 'Having pain or discomfort' and 'Doing usual activities' items were well understood. The examples of usual activities were found to be helpful. However, in some countries, one or two items led to misunderstanding, particularly the item covering the 'Looking after myself' domain. Four respondents from Spain (aged 8 to 9) thought the item referred to being able to provide for oneself (e.g. preparing food). In Italy, some respondents (five sick children and one healthy child) interpreted the item as referring to having to be careful because of their or others disease (being cautious, remembering to take medicines etc.). Two children from Spain and eight children from Italy reported problems with self-care because they had not yet learned to perform activities of personal hygiene. However, since the item was understood by the majority of respondents, it was left unchanged.

As regards specific problems in some language versions a change in wording was permitted, as long as the meaning of the item remained essentially unchanged. For example, the word 'difficulties' was found to be more appropriate than 'problems' in Sweden and Italy. If alternative translations were thought to be more difficult to understand, however, the original translation was retained. Thus, in Germany, the double negative included in the 'Feeling worried, sad or unhappy' dimension ('not unhappy') was retained despite criticism. 


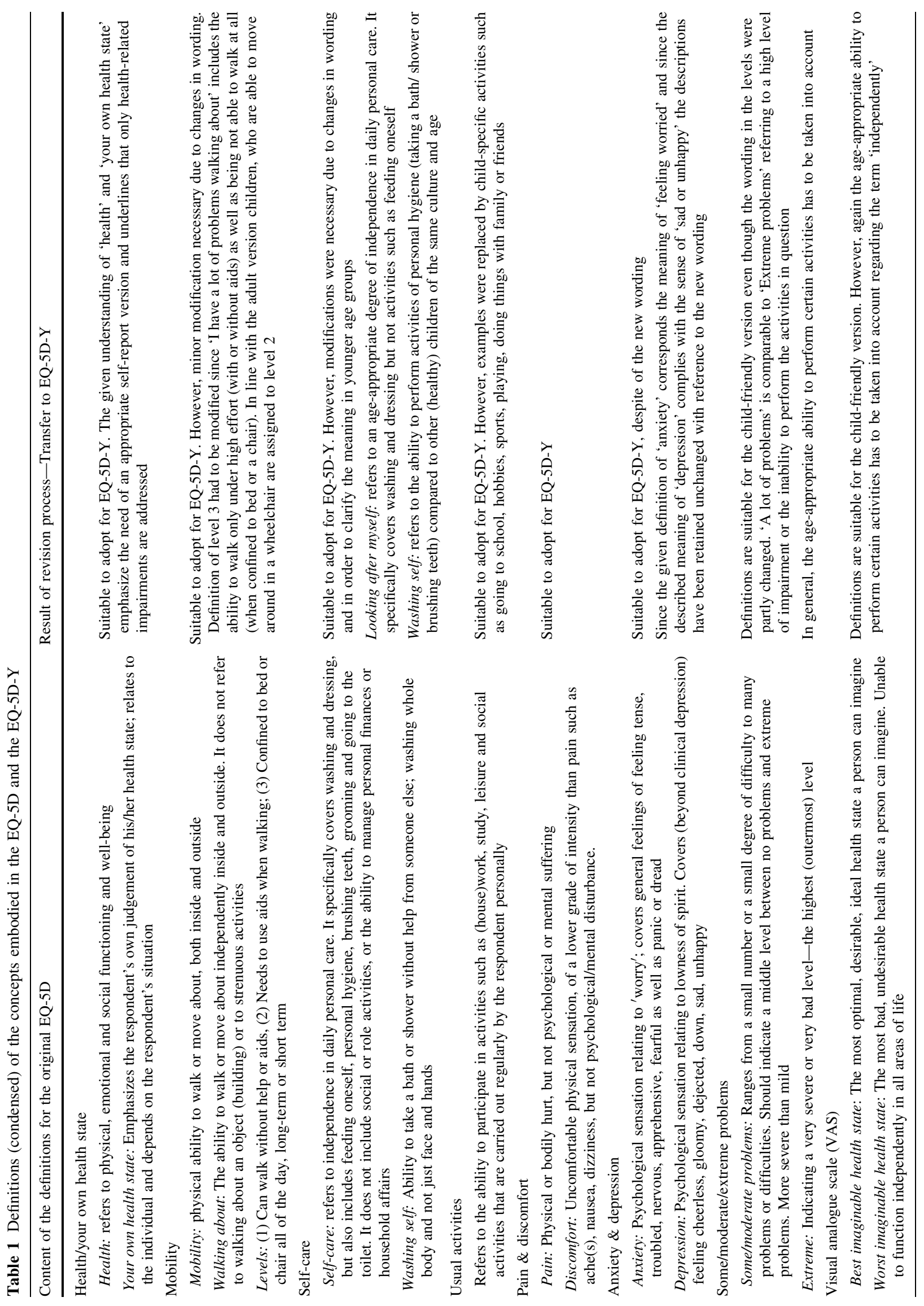


Table 2 Comparison of the EQ-5D and the EQ-5D-Y English language source version

\begin{tabular}{|c|c|}
\hline Original wording: standard adult EQ-5D & Modified wording: child-friendly EQ-5D-Y \\
\hline No heading & Describing your health TODAY \\
\hline $\begin{array}{l}\text { By placing a tick in one box in each group below, please indicate } \\
\text { which statements best describe your own health state today }\end{array}$ & $\begin{array}{l}\text { Under each heading, mark ONE box that best describes your } \\
\text { health TODAY }\end{array}$ \\
\hline Mobility & Mobility (walking about) \\
\hline I have no problems in walking about & I have no problems walking about \\
\hline I have some problems in walking about & I have some problems walking about \\
\hline I am confined to bed & I have a lot of problems walking about \\
\hline Self-care & Looking after myself \\
\hline I have no problems with self-care & I have no problems washing or dressing myself \\
\hline I have some problems washing or dressing myself & I have some problems washing or dressing myself \\
\hline I am unable to wash or dress myself & I have a lot of problems washing or dressing myself \\
\hline Usual activities (e.g. work, study, housework, family or leisure activities) & $\begin{array}{l}\text { Doing usual activities (for example, going to school, hobbies, } \\
\text { sports, playing, doing things with family or friends) }\end{array}$ \\
\hline I have no problems with performing my usual activities & I have no problems doing my usual activities \\
\hline I have some problems with performing my usual activities & I have some problems doing my usual activities \\
\hline I am unable to perform my usual activities & I have a lot of problems doing my usual activities \\
\hline Pain/discomfort & Having pain or discomfort \\
\hline I have no pain or discomfort & I have no pain or discomfort \\
\hline I have moderate pain or discomfort & I have some pain or discomfort \\
\hline I have extreme pain or discomfort & I have a lot of pain or discomfort \\
\hline Anxiety/depression & Feeling worried, sad or unhappy \\
\hline I am not anxious or depressed & I am not worried, sad or unhappy \\
\hline I am moderately anxious or depressed & I am a bit worried, sad or unhappy \\
\hline I am extremely anxious or depressed & I am very worried, sad or unhappy \\
\hline No heading & How good is your health TODAY \\
\hline \multirow{3}{*}{$\begin{array}{l}\text { To help people say how good or bad a health state is, we have drawn a } \\
\text { scale (rather like a thermometer) on which the best state you can } \\
\text { imagine is marked } 100 \text { and the worst state you can imagine } \\
\text { is marked } 0\end{array}$} & $\begin{array}{l}\text { We would like to know how good or bad your health is } \\
\text { TODAY. }\end{array}$ \\
\hline & This line is numbered from 0 to 100. \\
\hline & 100 means the best health you can imagine. \\
\hline \multirow{2}{*}{$\begin{array}{l}\text { We would like you to indicate on this scale how good or bad your } \\
\text { own health is today, in your opinion. Please do this by drawing } \\
\text { a line from the box below to whichever point on the scale indicates } \\
\text { how good or bad your health state is today }\end{array}$} & 0 means the worst health you can imagine. \\
\hline & $\begin{array}{l}\text { Please mark an } \mathrm{X} \text { on the line that shows how good or bad your } \\
\text { health is TODAY. }\end{array}$ \\
\hline Best imaginable health state & The best health you can imagine \\
\hline Worst imaginable health state & The worst health you can imagine \\
\hline
\end{tabular}

Table 3 Cognitive interview samples

\begin{tabular}{lllll}
\hline & Germany & Italy & Spain & Sweden \\
\hline Recruitment & Convenience sample & Convenience sample & Convenience sample & Convenience sample \\
$N$ & 8 & 37 & 20 & 38 \\
Gender & 4 boys/4 girls & 22 boys/15 girls & 10 boys/10 girls & 21 boys/17 girls \\
& 5 healthy, 3 chronically ill & 17 healthy, 20 chronically ill & 20 healthy children & 38 healthy children \\
Age range (years) & $8-16$ & $8-15$ & $8-18$ & $8-13$ \\
Mean age (SD) & $11.5(2.4)$ & $11.6(2.3)$ & $12.5(3.0)$ & $11.4(1.7)$ \\
Methods & $\begin{array}{l}\text { Paraphrasing, general } \\
\text { probing }\end{array}$ & Paraphrasing, free association, & Paraphrasing, general & General probing, free \\
& & numeric scale & probing, numeric scale & association \\
\hline
\end{tabular}


Regarding an adaptation of the VAS six different versions were tested in Italy and two different versions were tested in Germany. Some pupils had difficulty completing the standard adult EQ-5D VAS which requires the respondent to draw a line from a box in the centre of the page to the scale. In Italy and Germany, using a cross to rate their own health state directly on the VAS improved respondents' understanding of the task. Moreover, some adjustments in the instructions were found helpful in improving comprehensibility. Further, a comparison of different VAS versions in Italy showed that interviewees preferred a more detailed graduated VAS (specifying 0, 5, 10 , etc. instead of $0,10,20$, etc.) [37]. Based on these results, a modified VAS for younger respondents was developed which included simplified instructions and a more detailed graduated VAS [37].

In conclusion, the final consensus version was generally accepted by the interviewees. The descriptive system was completed by respondents in almost all cases without assistance. Although some modifications were made to other language versions to adapt the EQ-5D-Y to specific cultural contexts, there was no indication that amendments to the wording of the English language source version were required. Therefore, the descriptive system of the pilot questionnaire and the modified VAS were accepted as the final draft.

Frequencies of reported problems: comparison of the EQ-5D and the EQ-5D-Y

Table 4 shows the percentages of reported problems in the different domains as well as the mean VAS scores for respondents in Spain, Germany and South Africa. In all three countries, pupils tended to report more problems on the EQ-5D-Y 'Mobility (walking about)' $(P<0.05$ in Germany and South Africa), 'Having pain or discomfort' $(P<0.05$ in Germany and Spain) and 'Feeling worried, sad or unhappy' domains than in the EQ-5D $(P<0.05$ between the EQ-5D and EQ-5D-Y in all three countries). There was no consistent pattern of differences in responses on the 'Looking after myself' or 'Doing usual activities' domain.

The EQ-5D-Y also generally provided a more complete data set with fewer missing values compared to the EQ-5D. In Spain, differences for missing values between the two versions of the descriptive system were small and not statistically significant. In Germany, however, the EQ-5D$Y$ produced significantly lower percentages of missing values in all dimensions. In South Africa, a clear trend towards fewer missing values on the EQ-5D-Y was observed which was almost statistically significant for three of the five dimensions. On the VAS, there were noticeable differences in the proportion of missing values between the EQ-5D-Y and the EQ-5D (Table 5). In Germany, pupils also rated the comprehensibility of the version they received and we found that the EQ-5D-Y was generally considered easier to understand than the EQ-5D (data not shown).

\section{Discussion}

Our aim was to develop a modified version of the EQ-5D for use in respondents from 8 years onwards which includes the advantages of the standard adult EQ-5D. The development process of the EQ-5D-Y described in this paper was successful in deriving a modified, but nevertheless similar version of the EQ-5D through making ageappropriate adaptations.

The domains of the EQ-5D's descriptive system were found to be transferable to the population of children and adolescents. Although aspects such as mobility seem to be less important in the young population compared to adults, such dimensions were retained due to the instrument's generic character and necessity to be applicable also for sick patients.

In the revision of the wording, several difficulties were identified in the EQ-5D which required rephrasing to improve comprehensibility in the target population. Modifications were predominantly minor and did not affect item content. However, in some cases, rephrasing resulted in a deviation from the meaning of the EQ-5D which had to be reflected in the item definitions. A notable example was the use of less extreme response options in the third level of the EQ-5D-Y which refer to having considerable difficulty in performing an activity rather than being unable to perform it, as in the EQ-5D. Though this may limit the comparability of results with the EQ-5D, it was felt to be an acceptable trade-off given the expectation that the new version would lead to fuller use of response options across the whole range of severity.

The results of the cognitive interviews showed that the modified version was well accepted and comprehensible to young respondents. The main challenge with regard to validity was to clarify that the instrument only addresses health-related impairment and not age-related inability in performing certain activities. In a direct quantitative comparison with the standard adult EQ-5D, the EQ-5D-Y was judged to be easier to fill in and yielded fewer missing values. Likewise, in Germany and South Africa, there were markedly fewer missing values on the VAS used in the EQ5D-Y. Smaller differences between the two VAS versions in Spain might be attributable to an ordering effect, when children 'learned' to fill in the VAS in the EQ-5D-Y and then did it the same way in the EQ-5D at the end of the test battery. Further, using a different method than that 
Table 4 Percentages of reported problems in the EQ-5D-Y versus the EQ-5D (categories 'some' and 'a lot of' problems collapsed to calculate $\chi^{2}$ statistics)

\begin{tabular}{|c|c|c|c|c|c|c|}
\hline & \multicolumn{2}{|l|}{ Germany } & \multicolumn{2}{|l|}{ South Africa } & \multicolumn{2}{|l|}{ Spain } \\
\hline & EQ-5D-Y $(n=756)$ & EQ-5D $(n=730)$ & EQ-5D-Y $(n=258)$ & EQ-5D $(n=259)$ & EQ-5D-Y $(n=973)$ & EQ-5D $(n=973)$ \\
\hline \multicolumn{7}{|c|}{ Mobility (walking about) ${ }^{\mathrm{a}, \mathrm{b}}$} \\
\hline No & 92.3 & 95.5 & 87.4 & 93.0 & 95.3 & 96.7 \\
\hline Some & 7.6 & 3.8 & 12.3 & 5.8 & 4.6 & 2.9 \\
\hline A lot of & 0.1 & 0.7 & 0.4 & 1.2 & 0.1 & 0.4 \\
\hline \multicolumn{7}{|c|}{ Looking after myself ${ }^{b}$} \\
\hline No & 98.1 & 97.5 & 96.9 & 100 & 98.6 & 98.6 \\
\hline Some & 1.6 & 2.1 & 2.8 & 0.0 & 1.1 & 1.2 \\
\hline A lot of & 0.3 & 0.4 & 0.4 & 0.0 & 0.3 & 0.2 \\
\hline \multicolumn{7}{|c|}{ Doing usual activities ${ }^{\mathrm{a}}$} \\
\hline No & 93.5 & 87.3 & 84.3 & 86.1 & 93.7 & 92.8 \\
\hline Some & 6.2 & 11.7 & 15.0 & 13.5 & 5.7 & 6.8 \\
\hline A lot of & 0.3 & 1.0 & 0.8 & 0.4 & 0.6 & 0.4 \\
\hline \multicolumn{7}{|c|}{ Having pain or discomfort ${ }^{\mathrm{a}, \mathrm{c}}$} \\
\hline No & 62.5 & 72.1 & 52.8 & 60.3 & 80.0 & 85.5 \\
\hline Some & 36.2 & 26.2 & 45.5 & 38.5 & 19.0 & 7.4 \\
\hline A lot of & 1.3 & 1.7 & 0.8 & 1.2 & 1.0 & 0.5 \\
\hline \multicolumn{7}{|c|}{ Feeling worried, sad or unhappy ${ }^{a, b, c}$} \\
\hline Not & 60.1 & 78.0 & 61.4 & 72.7 & 76.9 & 92.5 \\
\hline A bit & 36.0 & 20.3 & 34.7 & 23.5 & 22.0 & 7.4 \\
\hline Very & 3.9 & 1.7 & 3.9 & 3.7 & 1.1 & 0.5 \\
\hline \multicolumn{7}{|l|}{ VAS } \\
\hline Mean & 83.7 & 83.5 & 77.3 & 74.6 & 83.8 & 83.8 \\
\hline SD & 16.0 & 16.8 & 15.7 & 16.1 & 13.7 & 14.2 \\
\hline Median & 90.0 & 90.0 & 80.0 & 79.5 & 90.0 & 85 \\
\hline Range & $8-100$ & $0-100$ & $34-100$ & $30-100$ & $20-100$ & $20-100$ \\
\hline Difference & $P=0.90$ & & $P=.067$ & & $P=206$ & \\
\hline
\end{tabular}

a Significant differences $(P<.05)$ between results of the two versions in Germany $\left(\chi^{2}\right.$ statistics $)$

b Significant differences $(P<.05)$ between results of the two versions in South Africa $\left(\chi^{2}\right.$ statistics)

${ }^{\mathrm{c}}$ Significant differences $(P<.05)$ between results of the two versions in Spain (Wilcoxon)

requested to score the VAS did not lead to the score being considered invalid (missing) in Spain, as long as the score was unambiguous.

The assumption that the frequency of respondents reporting more severe problems would increase due to the use of a less extreme upper severity level on the first three dimensions in the EQ-5D-Y was not borne out in current study, suggesting that the new wording might have little effect on frequency distributions on these dimensions. Nevertheless, the two versions should be tested side-byside in clinical samples with a wider range of problems before such a conclusion can be definitively drawn. In general, ceiling effects were smaller in the EQ-5D-Y since the respondents used the 'some problems' option more often. This was particularly the case in the last two dimensions ('having pain \& discomfort' and 'feeling worried, sad or unhappy') which address problems that are more common in children's lives than impairments in mobility, self-care or everyday activities.

Even though the different distribution of reported problems in both versions show that they cannot be used interchangeably, the EQ-5D-Y is still conceptually equivalent to the EQ-5D and provides an opportunity to compare children's and adult's ratings in corresponding dimensions. Given the apparent differences in distributions, particularly in the last two dimensions, future research should focus on the development of correction factors that will allow for comparison between the adult and youth versions.

The present study had some limitations. The fact that the EQ-5D-Y results from an adaptation of an existing instrument and not from an in-depth examination of the conceptualization, structure and content specific to children's 
Table 5 Percentages of missing values in the EQ-5D-Y compared to the EQ-5D

\begin{tabular}{|c|c|c|c|c|c|c|c|c|}
\hline \multicolumn{3}{|l|}{ Germany $^{a}$} & \multicolumn{3}{|c|}{ South Africa ${ }^{a}$} & \multicolumn{3}{|l|}{ Spain $^{\mathrm{b}}$} \\
\hline $\begin{array}{l}\text { EQ-5D-Y } \\
(n=756)\end{array}$ & $\begin{array}{l}\text { EQ-5D } \\
(n=730)\end{array}$ & $P$ & $\begin{array}{l}\text { EQ-5D-Y } \\
(n=258)\end{array}$ & $\begin{array}{l}\text { EQ-5D } \\
(n=259)\end{array}$ & $P$ & $\begin{array}{l}\text { EQ-5D-Y } \\
(n=973)\end{array}$ & $\begin{array}{l}\text { EQ-5D } \\
(n=973)\end{array}$ & $P$ \\
\hline \multicolumn{9}{|c|}{ Mobility (walking about) } \\
\hline 0.4 & 2.2 & .002 & 2.3 & 8.5 & .029 & 0 & 0.3 & 0.250 \\
\hline \multicolumn{9}{|c|}{ Looking after myself } \\
\hline 0.3 & 1.6 & .004 & 1.9 & 7.7 & .082 & 0 & 0.3 & 0.250 \\
\hline \multicolumn{9}{|c|}{ Doing usual activities } \\
\hline 0.3 & 1.8 & .002 & 1.9 & 7.3 & .056 & 0 & 0.3 & 0.250 \\
\hline \multicolumn{9}{|c|}{ Having pain or discomfort } \\
\hline 0.7 & 1.8 & .027 & 1.9 & 7.3 & .056 & 0 & 0.5 & 0.063 \\
\hline \multicolumn{9}{|c|}{ Feeling sad, worried or unhappy } \\
\hline 0.5 & 2.5 & .001 & 1.9 & 8.1 & .005 & 0 & 0.3 & 0.250 \\
\hline \multicolumn{9}{|l|}{ VAS } \\
\hline 8.9 & 16.7 & $<.001$ & 4.6 & 17.1 & $<.001$ & 3.0 & 5.0 & 0.004 \\
\hline
\end{tabular}

and adolescents' HRQOL might result in exclusion of aspects of HRQOL which are relevant to young respondents. However, the five health domains covered by the EQ-5D descriptive system accord with previous reviews about the main components of children and adolescents HRQOL [1, 41] and are covered by the most widely used HRQOL instruments specifically designed for young respondents. Nevertheless, further research will need to examine how far the addition of further domains might improve the performance of the instrument in children and adolescents. Another limitation is that the testing of the EQ-5D-Y was conducted predominantly in healthy samples which provide only limited information. Further research is needed to test the EQ-5D-Y in population-based and clinical subgroups. Likewise, the psychometric properties of the new version require assessment, and initial results on the EQ-5D-Y's feasibility, reliability and preliminary validity are published elsewhere [28].

Another crucial topic for further research is the use of the EQ-5D-Y in economic evaluations. Although it largely corresponds to the adult version, the EQ-5D-Y is a distinct instrument from the standard EQ-5D. Therefore, the existing EQ-5D social value sets cannot be assumed to be the appropriate preference weights. Any future valuation of health states generated by the EQ-5D-Y will have to address several very interesting issues, including the question of how social preferences should be elicited and who they should be elicited from. Clearly, demonstrating the appropriateness of the instrument for use in younger populations is an essential first step towards being able to include it as an outcome measure in economic evaluations in those populations. Hopefully, however, the availability of the EQ-5D-Y will help facilitate measurement of HRQOL in children and adolescents in a wide range of applications where social preference weights are not necessarily required.

Acknowledgments This work was financially supported and facilitated by the EuroQol Foundation. (The EuroQoL Foundation is a non-profit organization.) We thank Professor Magnus Svartengren, Karolinska Institutet, Sweden, Ph.Lic. Anna Lugnér, National Institute for Public Health and Environment, The Netherlands and RN Margareta Eriksson, Sachs' Children's Hospital, Sweden for participation in the development of the Swedish child-friendly pilot version of the EQ-5D instrument. We also thank the Italian team members: Carlo Tomasetto, Maria C Matteucci and Patrizia Selleri, from the Department of Educational Sciences and Serena Broccoli, from the Department of Statistics, University of Bologna; Barbara Pacelli, from the Epidemiology Unit, Local Health Authority, Bologna; Francesca Borghetti, Centre of Pharmacoeconomics, University of Milan, Milan, Italy. The EQ-5D-Y is a copyrighted instrument. All requests for EQ-5D-Y translations should be sent to the EuroQol Executive Office in Rotterdam, The Netherlands (userinformationservice@euroqol.org).

Open Access This article is distributed under the terms of the Creative Commons Attribution Noncommercial License which permits any noncommercial use, distribution, and reproduction in any medium, provided the original author(s) and source are credited.

\section{References}

1. Ravens-Sieberer, U., Erhart, M., Wille, N., Wetzel, R., Nickel, J., \& Bullinger, M. (2006). Generic health-related quality of life assessment in children and adolescents: Methodological considerations. Pharmacoeconomics, 24, 1199-1220.

2. Varni, J. W., Burwinkle, T. M., \& Lane, M. M. (2005). Healthrelated quality of life measurement in paediatric clinical practice: An appraisal and precept for future research and application. Health and Quality of Life Outcomes, 3, 34. 
3. Seid, M., Varni, J. W., Segall, D., \& Kurtin, P. S. (2004). Healthrelated quality of life as a predictor of pediatric healthcare costs: A two-year prospective cohort analysis. Health and Quality of Life Outcomes, 2, 48.

4. Rebok, G., Riley, A., Forrest, C., Starfield, B., Green, B., Robertson, J., et al. (2001). Elementary school aged children's report of their health: A cognitive interviewing study. Quality of Life Research, 10, 59-70.

5. Raat, H., Bonsel, G. J., Essink-Bot, M. L., Landgraf, J. M., \& Gemke, R. J. B. J. (2002). Reliability and validity of comprehensive health status measures in children: The Child Health Questionnaire in relation to the Health Utilities Index. Journal of Clinical Epidemiology, 55, 67-76.

6. Eiser, C., \& Morse, R. (2001). A review of measures of quality of life for children with chronic illness. Archives of Disease in Childhood, 84, 205-211.

7. Mansour, M. E., Kotagal, U., Rose, B., Ho, M., Brewer, D., RoyChaudhury, A., et al. (2003). Health-related quality of life in urban elementary schoolchildren. Pediatrics, 111, 1372-1381.

8. Blair, M. (2000). Public health: Taking a population perspective on child health. Archives of Disease in Childhood, 83, 7-9.

9. Verrips, E. G. H., Vogels, T. G. C., Koopman, H. M., Theunissen, N. C. M., Kamphuis, S. R. P., Fekkes, M., et al. (1999). Measuring health-related quality of life in a child population. European Journal of Public Health, 9, 188-193.

10. Matza, L. S., Swensen, A. R., Flood, E. M., Secnik, K., \& Leidy, N. K. (2004). Assessment of health-related quality of life in children: A review of conceptual, methodological, and regulatory issues. Value in Health, 7, 79-92.

11. Gemke, R. J. (1995). Comparative assessment of pediatric intensive care: A national multicenter study. Critical Care Medicine, 23, 238-245. (on behalf of the Pediatric Intensive Care Assessment of Outcome (PICASSO) Study Group).

12. Detmar, S. B., Bruil, J., Ravens-Sieberer, U., Gosch, A., \& Bisegger, C. (2006). The use of focus groups in the development of the KIDSCREEN HRQL Questionnaire. Quality of Life Research, 15, 1345-1353.

13. Petersen, C., Schmidt, S., Power, M., \& Bullinger, M. (2005). Development and pilot-testing of a health-related quality of life chronic generic module for children and adolescents with chronic health conditions: A European perspective. Quality of Life Research, 14, 1065-1077.

14. Bruil, J., Maes, S., le Coq, L., \& Boeke, J. (1996). The development of the how are you (HAY), a quality of life questionnaire for children. Quality of Life Newsletter, 13, 9.

15. Ravens-Sieberer, U., Gosch, A., Rajmil, L., Erhart, M., Bruil, J., Power, M., et al. (2007). The KIDSCREEN-52 quality of life measure for children and adolescents: Psychometric results from a cross-cultural survey in 13 European countries. Value in Health, $11,645-658$.

16. Sapin, C., Simeoni, M. C., El Khammar, M., Antoniotti, S., \& Auquier, P. (2005). Reliability and validity of the VSP-A, a health-related quality of life instrument for ill and healthy adolescents. Journal of Adolescent Health, 36, 327-336.

17. Landgraf, J. M., Maunsell, E., Speechley, K. N., Bullinger, M., Campbell, S., Abetz, L., et al. (1998). Canadian-French, German and UK versions of the Child Health Questionnaire: Methodology and preliminary item scaling results. Quality of Life Research, 7, 433-445.

18. Starfield, B., Riley, A., Ensminger, M., Green, B., Ryan, S., KimHarris, S., et al. (1994). Manual for the child health and illness profile-adolescent edition (CHIP-AE). Baltimore, MD: The John Hopkins University.

19. Varni, J. W., Seid, M., \& Rode, C. A. (1999). The PedsQLTM: Measurement model for the pediatric quality of life inventory. Medical Care, 37, 126-139.
20. Rabin, R., \& de Charro, F. (2001). EQ-5D: A measure of health status from the EuroQol Group. Annals of Medicine, 33, 337-343.

21. Hennessy, S., \& Kind, P. (2002). Measuring health status in children: Developing and testing a child-friendly version of EQ5D. In Proceedings of the 19th plenary meeting of the EuroQol Group. York, United Kingdom.

22. Stolk, E. A., Busschbach, J. J., \& Vogels, T. (2000). Performance of the EuroQol in children with imperforate anus. Quality of Life Research, 9, 29-38.

23. Badia, X., Gracia-Hernandez, G., Cobos, N., López-David, C., Nocea, G., Roset, M., et al. (2001). Validity of the Spanish version of the Pediatric Quality of Life Questionnaire for evaluating quality of life in asthmatic children. Clinical Medicine, 116, 565-572.

24. Burström, K., Egmar, A.-C., Eriksson, M., Lugnér, A., \& Svartengren, M. (2003). Development of a Swedish child-friendly version of the EQ-5D. Paper presented at the 20th plenary meeting of the EuroQol group. Bled, Slovenia.

25. Burström, K., Egmar, A.-C., Eriksson, M., \& Svartengren, M. (2005). EQ-5D in a Swedish representative population survey of children aged 8 and 12 years: A child-friendly version used in a clinical examination. In Proceedings of the 22nd plenary meeting of the EuroQol Group. Oslo, Norway.

26. Jelsma, J., Knight, F., Meyer, L., et al. (2005). The validity of the UK "child friendly" version of the EQ-5D in South African children aged 9 to 12 years. In Proceedings of the 22nd plenary meeting of the EuroQoL Group. Oslo, Norway.

27. Jelsma, J., Coppez, P., Jehosofat, T., et al. (2006). The validity of the child friendly version of the EQ-5D when used with high school English speaking children in South Africa-preliminary results. In Proceedings of the 23rd plenary meeting of the EuroQoL Group. Barcelona, Spain.

28. Ravens-Sieberer, U., Wille, N., Badia, X., Bonsel, G., Burström, K., Cavrini, G., et al. Feasibility, reliability, and validity of the EQ-5D-Y: Results from a multinational study. Quality of Life Research. doi:10.1007/s11136-010-9649-x.

29. Szende, A., Oppe, M., \& Devlin, N. (2007). EQ-5D valuation sets: Inventory, comparative review and user guide. Rotterdam: Springer.

30. Fox-Rushby, J., \& Selai, C. (2003). What concepts does the EQ5D measure? Intentions and interpretations. In R. Brooks, R. Rabin, \& F. de Charro (Eds.), The measurement and valuation of health status using EQ-5D: A European Perspective (pp. 167182). Dordrecht: Kluwer.

31. Herdman, M., Fox-Rushby, J., Rabin, R., Badia, X., \& Selai, C. (2003). Producing other language versions of the EQ-5D. In R. Brooks, R. Rabin, \& F. de Charro (Eds.), The measurement and valuation of health status using EQ-5D: A European Perspective (pp. 183-190). Dordrecht: Kluwer.

32. Forsyth, B. H., \& Lessler, J. T. (1991). Cognitive laboratory methods: A taxonomy. In P. Biemer, R. Groves, L. Lyberg, et al. (Eds.), Measurement errors in surveys (pp. 393-418). New York: Wiley.

33. Conrad, F., Blair, J., \& Tracy, E. (1999). Verbal reports are data! A theoretical approach to cognitive interviews. FCSM conference.

34. Prüfer, P., \& Rexroth, M. (2000). Zwei-Phasen-Pretesting. ZUMA-Arbeitsbericht 2000/08, Mannheim.

35. Cull, A., Sprangers, M., Bjordal, K., Aaronson, N., West, K., \& Bottomley, A. (2002). EORTC quality of life group translation procedure (2nd ed.) Brussels (on behalf of the EORTC Quality of Life Group).

36. Doise, W., Clemence, A., \& Lorenzi-Cioldi, F. (1993). The quantitative analysis of social representations. New York: Harvester Wheatsheaf.

37. Scalone, L., Tomasetto, C., Matteucci, M.C., Selleri, P., Broccoli, S., Pacelli, B. et al. Development and validation of EQ-5D to assess health related quality of life in children and adolescents: 
The contribution of the Italian EQ-5D-Y. Quality of Life Research (submitted).

38. Burström, K., Egmar, A.-C., Lugnér, A., Eriksson, M., \& Svartengren, M. (2010). A Swedish child-friendly pilot version of the EQ-5D instrument - the development process. European Journal of Public Health. doi:10.1093/eurpub/ckq037.

39. Burström, K., Svartengren, M., \& Egmar, A.-C. (2010). Testing a Swedish pilot version of the EQ-5D instrument-initial results. European Journal of Public Health. doi:10.1093/eurpub/ckq042.
40. Jelsma, J. A comparison of the EQ-5D and the EQ-5D-Y health related quality of life instruments in South African children. International Journal of Rehabilitation Research (accepted for publication).

41. Rajmil, L., Herdman, M., Fernandez de Sanmamed, M. J., Detmar, S., Bruil, J., Ravens-Sieberer, U., et al. (2004). Generic health-related quality of life instruments in children and adolescents: A qualitative analysis of content. Journal of Adolescent Health, 34, 37-45. 